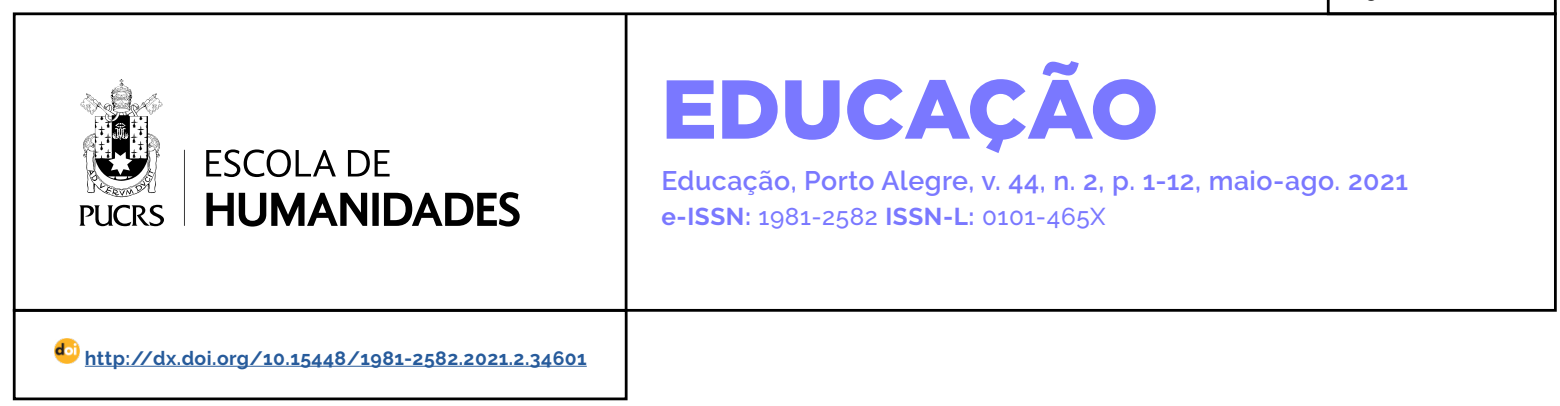

SEÇÃO: OUTROS TEMAS

\title{
Instrumento de pesquisa para prevenção da evasão nas instituições de ensino superior à distância: metodologia de construção
}

\author{
Instrument of research for evasion prevention at distance higher education institutions: \\ construction methodology \\ Instrumento de investigación para prevención de la evasión en las instituciones de \\ enseñanza superior a distancia: metodología de construcción
}

\section{Oberdan Santos da \\ Costa ${ }^{1}$ \\ orcid.org/0000-0002-2448-5247 \\ sc.oberdan@gmail.com}

Luis Borges Gouveia ${ }^{1}$

orcid.org/0000-0002-2079-3234

Imbg@ufp.edu.pt

\section{Luis Simoes da Cunha ${ }^{2}$} orcid.org/0000-0001-8772-6944 luiscunha@ismt.pt

Recebido em: 27 jun. 2019. Aprovado em: 27 mai. 2021. Publicado em: 16 set. 2021.
Resumo: O objetivo deste estudo é relatar as etapas de construção do instrumento de pesquisa para prevenção da evasão, nas instituições de ensino superior à distância. O instrumento foi construído com a finalidade de coletar informações sobre o apoio acadêmico, recebido pelos estudantes, às mudanças nas circunstâncias da vida do estudante durante o processo de formação, aspectos motivacionais, experiência de aprendizagem em geral e características individuais. No estudo foi adotada uma abordagem quantitativa, com base em estudos nacionais e internacionais. Os resultados apontaram $83.3 \%$ de concordância entre os juizes para os constructos da motivação, apoio (tutor, familiar e financeiro) e 100\% de concordância para os constructos sexo, idade, educação anterior e situação de trabalho. Esses dados podem ser úteis aos pesquisadores e estudiosos para o alinhamento do foco e do objetivo de seus estudos.

Palavras-chave: metodologia, instrumento, evasão, educação superior, educação à distância.

Abstract: The instrument was constructed to gather information about the academic support received by students, changes in the circumstances of the student's life during the training process, motivational aspects, general learning experience and individual characteristics. The study adopts a quantitative approach based on national and international studies. The objective of the study is to report on the stages of construction of the research tool to prevent evasion in Higher Education Institutions at a distance. The results indicate 83.3\% concordance between the judges for the motivational, support (tutor, family and financial) constructs and $100 \%$ agreement for the constructs sex, age, previous education and work situation. These may be useful to researchers and scholars for aligning the focus and purpose of their studies.

Keywords: methodology, construction, instrument, evasion, higher education, distance education.

Resumen: El instrumento fue construido con el fin de recoger informaciones sobre el apoyo académico recibido por los estudiantes, los cambios en las circunstancias de la vida del estudiante durante el proceso de formación, aspectos motivacionales, experiencia de aprendizaje en general y caracteristicas individuales. El estudio adopta un enfoque cuantitativo basado en estudios nacionales e internacionales. El objetivo del estudio es relatar las etapas de construcción del instrumento de investigación para prevenir la evasión en las Instituciones de Enseñanza Superior a distancia. Los resultados apuntan el 83.3\% de concordancia entre los jueces para los constructos de la motivación, apoyo (tutor, familiar y financiero) y el 100\% de concordancia para los constructos sexo, edad, educación anterior y situación de trabajo. Estos pueden ser útiles a los investigadores y estudiosos para la alineación del foco y del objetivo de sus estudios.

Palabras clave: metodologia, construcción, instrumento, evasión, educación superior, educación a distancia. 


\section{Introdução}

Alguns estudiosos sugerem que desistir é um processo, não um evento e, como resultado, pode ser causado por uma combinação de fatores (Mansfield, O'leary \& Webb, 2011). Nesse sentido, muitos têm sido os estudiosos a debruçarem-se sobre a problemática desse fenômeno, procurando desenvolver instrumentos de diferentes abordagens. Diante desse cenário, fez-se uma análise de conteúdo de estudos específicos de medidas, de modo a estabelecer um alinhamento no desenvolvimento do instrumento de pesquisa, com a estrutura do modelo conceitual desenvolvida com base nos modelos de Bean e Metzner (1985) e Park (2007), além dos trabalhos de Costa e Gouveia (2019) e Lee e Choi (2011). Esses últimos permitiram identificar as variáveis preditoras que determinam ou contribuem para o risco da evasão de estudantes na modalidade de educação à distância (EaD).

$O$ instrumento de pesquisa neste estudo foi construido com a finalidade de coletar informações sobre os fatores que afetam a decisão do comportamento de abandonar os estudos de discentes do ensino superior à distância. Nesse sentido, dirigimos o foco para o apoio acadêmico recebido pelos estudantes, as mudanças nas circunstâncias da vida durante o processo de formação universitária, os aspectos motivacionais, experiência de aprendizagem em geral e suas caracteristicas individuais.

O presente estudo tem como objetivo relatar as etapas de construção do instrumento de pesquisa para prevenção da evasão nas instituições de educação superior (IES), que oferecem programas de graduação na modalidade de ensino à distância. A formulação dos itens foi baseada em artigos nacionais e internacionais, recuperados de bases de dados digitais do Google Scholar e do Education Resources Information Center (ERIC), bem como na experiência dos autores na área da educação. Os 124 itens provenientes de várias fontes autorizadas foram avaliados por seis juizes, que, juntos, somam aproximadamente 90 anos de experiência na área da EaD.
A razão da construção desse instrumento de pesquisa é o fato de integrar um conjunto de dimensões, que são objetos da investigação do trabalho de doutoramento de um dos autores. Os resultados do estudo apontam $83,33 \%$ de concordância entre os juizes para os constructos "motivação do fator psicológico", "apoio tutor do fator acadêmico", "apoio familiar e apoio financeiro do fator ambientes". Houve 100\% de concordância entre os juizes para os constructos "sexo", "idade", "educação anterior" e "situação de trabalho/nº de horas trabalhada do fator caracteristica de fundo".

\section{Referencial teórico}

Estudos nacionais e estrangeiros no campo da educação superior têm direcionado seus esforços na busca de entender os motivos da evasão de estudantes. Alguns desses estudos concentram-se na construção da escala de instrumentos com foco na satisfação (Diniz \& Almeida, 2005; Schleich, Polydoro \& Santos, 2006), Escala de Interação com Pares para estudantes do ensino superior (Fior, Mercuri \& Almeida, 2011), envolvimento acadêmico (Fior, Mercuri \& Silva, 2013) e motivos para evasão do ensino superior (Ambiel, 2015).

Em seus estudos, Diniz e Almeida (2005) apresentam o processo de construção e de validação da Escala de Integração Social no Ensino Superior (EISES). Para os autores, as preocupações iniciais dos estudantes reportaram-se, principalmente, ao bem-estar pessoal e ao estabelecimento de novas relações, dimensões da satisfação consigo e com os outros (estrutura fatorial bidimensional hierárquica). Essas dimensões e o equilibrio emocional (estrutura fatorial tridimensional hierárquica) representam as áreas problemáticas da integração social dos discentes.

Alinhados ao foco da satisfação, Schleich, Polydoro e Santos (2006) analisaram os parâmetros psicométricos da Escala de Satisfação com a Experiência Acadêmica (ESEA). Para os autores, a falta de instrumentos nacionais devidamente validados para a avaliação da satisfação acadêmica justificou o desenvolvimento do estudo. Os autores 
afirmam, ainda, que conhecer as satisfações ou insatisfações dos estudantes auxilia a elevar o entendimento do impacto do ensino superior no desenvolvimento integral, já que o desencontro entre a diversidade de expectativas dos discentes e o que realmente a instituição oferece pode gerar baixo desempenho, reduzida integração, insucesso e, até mesmo, o abandono do curso.

Fior, Mercuri e Almeida (2011) apresentam, em seus estudos, a construção e as evidências de validade da Escala de Interação com Pares para estudantes do ensino superior. Os autores destacam que as relações que os alunos estabelecem com os pares são importantes para a aprendizagem e o desenvolvimento psicossocial e cognitivo. Também, acrescentam que o estudo psicométrico do instrumento permite afirmar que a Escala de Interação com os Pares apresenta os requisitos que possibilitam considerá-la como um instrumento adequado e fidedigno. Contudo, é pertinente que se realizem outros estudos, principalmente com estudantes de ensino superior que apresentam caracteristicas distintas das apresentadas pelos participantes do estudo de Fior, Mercuri e Almeida (2011), a fim de aprimorar os resultados psicométricos da escala.

Em seus estudos sobre evidências de validade da Escala de Envolvimento Acadêmico para universitários, Fior, Mercurie Silva (2013) afirmam que envolvimento acadêmico, entendido como a participação dos estudantes nas tarefas educativas, é imprescindivel para a aprendizagem, desenvolvimento e permanência no sistema educacional. Eles acrescentam, ainda, que a construção da escala contribui para ampliação do conhecimento sobre a vivência acadêmica e reafirma a diversidade de experiências presentes na formação universitária.

Ambiel (2015) relata, em seu estudo, a construção dos itens e validação da estrutura interna da escala de motivos para evasão do Ensino Superior. O autor observa dois pontos importantes na construção do instrumento. O primeiro destaca que a formulação dos itens se baseou em artigos brasileiros, recuperados de bases de dados digitais, bem como na vivência de estudantes de ensino superior ativos e evadidos. O segundo destaca que a construção da Escala de Motivos para Evasão do Ensino Superior (M-ES) não se baseou em uma teoria especifica, mas sim em motivos levantados junto a estudantes do ensino superior, juizes com experiência no assunto e em diversos estudos brasileiros, que, de forma independente, buscaram identificá-los.

Embora haja uma quantidade razoável de pesquisas nacionais e internacionais avaliando fatores e variáveis relacionadas à evasão, não foram encontrados na literatura instrumentos padronizados que afiram, especificamente, os motivos potenciais sobre o que afeta a decisão do comportamento de abandonar o curso de estudantes do ensino superior à distância.

Muitos dos estudos, observados para construção de instrumento para esta pesquisa, concentram sua atenção em somente um aspecto: notadamente, a satisfação tem sido objeto de várias pesquisas. Esses estudos de construção da escala contribuem para ampliação do conhecimento sobre a vivência na graduação e reafirmam a diversidade de experiências presentes durante a formação universitária.

Entende-se que a identificação dos itens potenciais para prever a evasão de estudantes importa para o planejamento de ações institucionais, acompanhamento e monitoramento sistêmico. Nesse sentido, o presente estudo tem como objetivo relatar as etapas de construção do instrumento de pesquisa para prevenção da evasão nas IES, que oferecem programas de graduação na modalidade de ensino à distância.

\section{Metodologia}

O estudo adota uma abordagem do tipo quantitativa. Quanto aos fins, esta é uma pesquisa descritiva, pois busca descrever os procedimentos metodológicos da construção de um instrumento de pesquisa para prevenção da evasão de estudantes na educação superior à distância. O detalhamento da construção do instrumento, a seguir, oferece as etapas do processo com seus respectivos fatores, fontes autorizadas, constructos e itens. 


\section{Construção do instrumento}

Apesar das complexidades inerentes ao fenômeno evasão de estudantes da EaD, o instrumento usado nesta pesquisa foi construído com a finalidade de coletar informações sobre o que afeta a decisão de abandonar o curso de estudantes dessa modalidade de ensino. Nesse sentido, o foco é dirigido para o apoio acadêmico recebido pelos discentes, as mudanças nas circunstâncias da vida durante o processo de formação, os aspectos motivacionais, experiência de aprendizagem em geral e caracteristicas individuais. Esses representam fatores de naturezas distintas, que influenciam o comportamento que levam a evasão de estudantes no ensino superior à distância. Etapas do processo da construção do instrumento de pesquisa foram adaptadas do estudo de Jun (2005) e estão detalhadas no Quadro 1.

Quadro 1 - Etapas do processo da construção do instrumento.

\begin{tabular}{|l|l|}
\hline Etapa & \multicolumn{1}{|c|}{ Processo } \\
\hline 1 & Clarificação do conceito \\
\hline 2 & Identificação de itens \\
\hline 3 & Tradução e construção \\
\hline 4 & Refinamento dos conjuntos de itens \\
\hline 5 & $\begin{array}{l}\text { Revisão pré-piloto do instrumento de } \\
\text { pesquisa }\end{array}$ \\
\hline
\end{tabular}

Fonte: Adaptado de Jun (2005).

\section{Clarificação do conceito}

Este estudo busca construir um instrumento de coleta de informações sobre o que afeta a decisão de estudantes do ensino superior à distância de abandonar os estudos e, posteriormente, o modelo conceitual com fatores para prever a evasão desses estudantes, com base numa revisão nos conceitos destacados neste artigo. A título de exemplos, tem-se os conceitos de Brown (1996), sobre o apoio tutor, OLC (2015) e Shelton (2011), sobre o apoio institucional dos fatores acadêmicos, Bean e Metzner (1985) e Lee e Choi (2011), sobre o apoio familiar, Bean e Metzner (1985) e Parker (1999), sobre apoio finan- ceiro e ambiente, Keller (1987, 2010) - modelo ARCS - e Balaban-Sali (2008), sobre a motivação e Parker (1999), sobre o lócus de controle dos fatores psicológicos. Esses conceitos são apresentados a seguir:

a) Apoio tutor: tem se mostrado importante na prevenção da evasão de estudantes de cursos de graduação na modalidade de ensino à distância, pois é relevante no contato inicial com o discente e em gerar um senso de comunidade na turma de estudos.

b) Apoio institucional: é um apoio essencial nos cursos à distância, em função da contribuição por meio de recursos tecnológicos e infraestrutura administrativa centralizada.

c) Apoio Familiar: diz respeito ao nivel de apoio que os alunos recebem de seus familiares e amigos para concluir o programa de curso.

d) Apoio de trabalho/organização: diz respeito a uma diferença de pressão para trabalhar mais horas ou mudanças nas responsabilidades de trabalho, que afetam o estudo.

e) Apoio Financeiro: refere-se ao ingresso e à permanência do estudante na IES. Quando o aluno não consegue pagar o curso em função de enfrentar dificuldades financeiras profundas, vê-se obrigado a abandoná-lo.

f) Atenção: desperta e sustenta a curiosidade e o interesse dos estudantes durante a instrução, por meio de uma variabilidade de eventos inesperados e situações incompativeis, que direcionam a atenção a estímulos apropriados.

g) Relevância: estratégias que apoiam as necessidades considerando a filiação, ou realização de interesses dos discentes, incluindo orientação, objetivo, correspondência motriz e familiaridade.

h) Confiança: fornece senso de autoestima, expectativa positiva e capacidade de sucesso em tarefas desafiadoras. Diferenças na confiança podem influen- 
ciar a permanência e a realização do estudante.

i) Satisfação: apoia os esforços dos alunos e faz com que sintam-se bem sobre suas realizações. Incluem reforço intrinseco, recompensas extrinsecas e equidade.

j) Lócus de controle: percepção de um indivíduo sobre o que causa ou influencia os seus resultados.

k) Evasão: diz respeito ao estudante que, após matriculado, abandona o seu programa de curso de graduação, a qualquer momento durante o seu desenvolvimento, e nunca retorna.

\section{Identificação de itens}

Nessa etapa, a recuperação da literatura foi realizada através do uso de recursos de biblioteca on-line, para o levantamento de trabalhos de teses, dissertações e artigos científicos na literatura nacional e internacional. As bases de dados pesquisadas incluíram o Google Scholar e o ERIC. Os seguintes termos-chave, que se encaixam nos domínios deste estudo, foram utilizados para definir o fundamento desta revisão de literatura: foram empregadas todas as combinações e permutações de EaD, evasão, preditor, modelo ARCS, abandono, dropout, fatores caracteristicas de fundo, acadêmicos, ambientes e psicológicos, motivação e lócus de controle. O resultado da revisão identificou 124 itens, provenientes das fontes autorizadas de Holder (2007) e Jun (2005); pesquisas adaptadas de Huang e Hew (2016), Keller (2010), Levy (2007), Loorbach et al. (2015), Park e Choi (2009) e Su (2016).

\section{Tradução e construção}

Boa parte dos itens identificados são de autores estrangeiros. Assim, fez-se necessária a tradução de alguns itens, originalmente no idioma inglês, para o português. Fizemos o processo de tradução usando os tradutores do Google, Microsoft e Iris Pen Executive 7, na tradução inicial. Como base, utilizamos Prieto (1992), que reco- menda algumas diretrizes para os procedimentos de tradução: (1) empregar palavras e frases com frequência semelhante de uso nos dois idiomas; (2) considerar as caracteristicas únicas do público pretendido; (3) utilizar os serviços de um revisor não familiarizado com o público pretendido, para identificar discrepâncias e fornecer uma verificação da inteligibilidade do texto alvo; (4) conduzir testes piloto da tradução, por meio da administração do instrumento aos membros da audiência pretendida. Após tradução inicial, contou-se com a experiência profissional de dois estudantes de doutorado da Universidade Fernando Pessoa (UFP) para ajudar no procedimento da tradução, identificar discrepâncias e fornecer uma verificação da inteligibilidade do texto alvo. Antes de iniciar o procedimento de tradução, os discentes da UFP receberam informações explícitas sobre o uso e a intenção do instrumento, bem como as diretrizes da tradução citadas anteriormente.

Depois do procedimento da tradução de itens, o instrumento foi encaminhado para o conselho de ética da UFP, para fins de parecer. Após parecer favorável pelo conselho de ética da UFP, iniciou-se o processo de construção do instrumento, através de um convite a 22 juizes especialistas da área da educação por meio do envio de um e-mail. Os seguintes critérios de inclusão e exclusão para participação dos especialistas no estudo foram: possuir titulação de doutor e/ ou mestre na área da educação e experiência profissional (ensino ou pesquisa) minima de cinco anos. O convite aos juizes para participar da construção do instrumento intitulado "apoio para o desenvolvimento do instrumento de pesquisa" contém quatro fatores preditores da evasão de estudantes no ensino à distância, ou seções (características de fundo, acadêmicos, ambientes e psicológicos), treze constructos e 124 itens para serem analisados e classificados pelos juizes, quando da representação e relevância para o objetivo da pesquisa.

O convite, encaminhado aos juizes para apoio ao desenvolvimento do instrumento de pesquisa, levou em consideração dois pontos importantes. o primeiro diz respeito à validade de conteúdo. 
Para garantir tal preceito, foram utilizadas várias fontes autorizadas para identificar itens de instrumentos de pesquisa (Perdue, 1999). Quatro seções de itens de escala e subescala foram identificados, com base em diversas pesquisas na área de EaD. O segundo item diz respeito à experiência dos juizes no campo da EaD. Dos 22 juizes, seis responderam à solicitação no prazo especificado, sendo um mestre, um doutorando e quatro doutores. Esses juizes, juntos, somam, aproximadamente, 90 anos de experiência na área. Considerando um critério de relevância, ou melhor, de representação dos itens para o constructo, houve, aproximadamente, 83,33\% de concordância entre os juizes para motivação subscala-atenção, subscala-relevância, subscala-satisfação e suscala-confiança da seção 3 - psicológicos. O constructor apoio tutor (seção-acadêmico) no criterio de relevância obteve 83.33\% de concorãncia entre os juizes. No critério representação, houve 83,33\% de concordância entre os juizes para os constructos apoio fa- miliar, apoio da organização e apoio financeiro (seção-ambiente) e 100\% de concordância para os constructos sexo, idade, educação anterior e situação de trabalho $/ \mathrm{n}^{\circ}$ de horas trabalhada (seção-característica de fundo). Diante das resposta dos juízes, foram excluídos os constructos "apoio da organização" e "lócus de controle", por não haver concordâncias suficientes, e foram mantidos os demais constructos, inicialmente propostos, em função das concordâncias.

Após análise e identificação dos itens do instrumento da pesquisa pelos juizes, resultou em 22 itens, de um total de 124 indicadores de informações, sobre o que afeta a decisão do estudante abandonar ou permanecer em seus estudos na modalidade de ensino à distância, e 11 constructos. Tais itens identificados deram origem ao questionário/instrumento utilizado nesta pesquisa, os quais foram resumidos, utilizando as fontes autorizadas, e distribuidos numericamente nos quatro constructos deste estudo, conforme Tabela 1.

TABELA 1 - Resumo das fontes autorizadas, constructos e itens do instrumento.

\section{APOIO ACADÊMICO}

\begin{tabular}{ll}
\hline Pesquisador & 1 \\
Total & $\mathbf{1}$ item
\end{tabular}

\section{APOIO FAMILIAR}

Holder (2007)

Total

\section{APOIO FINANCEIRO}

Holder (2007)

Total

\section{MOTIVAÇÃo}

Jun (2005)

Huang e Hew (2016) Adaptado de Keller (2010)

Loorbach et al. (2015)

Koller (2010) Course Interest Survey (CIS)
1

1 item

Subescala da motivação

Atenção Relevância Confiança Satisfação

1

2

0

\section{o}

1

1

2
$1 \quad 0$

$2 \quad 2$

$0 \quad 0$

$1 \quad 1$ 


\begin{tabular}{lll} 
Levy (2007) & 0 & 0 \\
Pesquisa adaptada de Levy (2007) & 0 & 4 \\
Subtotal & 3 & $\mathbf{1 4}$ itens \\
Total & Sexo - 1 \\
& $\mathbf{1}$ item \\
& Idade - 1 \\
Pesquisador & $\mathbf{1}$ item \\
& Educação anterior - 1 \\
& $\mathbf{1}$ item \\
Su (2016) & Carga de trabalho - 1 \\
& $\mathbf{1}$ Item \\
Total & $\mathbf{4}$ itens \\
\hline Total geral & $\mathbf{2 2}$ itens
\end{tabular}

Fonte: Elaborado pelos autores (2019).

Após apresentação dos constructos e itens identificados pelos juizes, observa-se que houve uma produção incisiva nos resultados. Os resultados trazem uma abordagem dos constructos, em que as instituições têm ou não controle, mas que podem, potencialmente, afastar o estudante da instituição. Mediante a essa observação, considera-se que as instituições têm controle sobre os constructos acadêmico e psicológico, mas, por outro lado, não têm controle sobre os constructos características de fundo e ambiente. Importa essa observação, pois guia o direcionamento de esforços das instituições, bem como caminhos para o refinamento de itens para o pesquisador.

\section{Refinamento e aperfeiçoamento do conjunto de itens}

Antes de iniciar-se o refinamento de itens, destaca-se a experiência dos pesquisadores na área de EaD, especificamente na gestão de polo educacional, bem como suas relações com gestores, coordenadores e consultores de instituições e polos de EaD. O refinamento de itens ocorrerá em três momentos. No primeiro momento do refinamento de itens, verifica-se os 22 potenciais, com o objetivo de revisar cuidadosamente os elementos, para clareza de redação, suprimir duplicações e lógica de classificação. Cada item foi revisado, para facilitar a compreensão, consistência na redação e evitar coloquialismos acadêmicos, utilizando classificação adequada de cada item nas escalas e subescalas a serem usadas. Mantendo-se a linha incisiva dos juizes, ofertam-se as seguintes questões gerais de discussão: "Para quem a pesquisa será aplicada?", "Em quais cursos superiores EaD a pesquisa será aplicada?", "Quando a pesquisa será aplicada?" e "Qual é o propósito da escala que será desenvolvida?". Completada essa tarefa, corrige-se a redação das questões impróprias de itens, em cada constructo, e relaciona-se cada questão com o ambiente da pesquisa.

No segundo momento, altera-se o nome do constructo "apoio tutor" para "apoio acadêmico", com o objetivo de coletar informações do tutor e da instituição em separados. De acordo com Mill (2012), a figura do tutor é tão importante para a relação ensino-aprendizagem, que o aluno reconhece nele (tutor) a própria instituição de ensino - dada a estreita relação entre eles. Em função de ambos terem responsabilidades diferentes no processo 
acadêmico, é importante que a informação a respeito do tutor e instituição sejam representadas em separado. Além dessa alteração, foi realizada uma nova numeração para a sequência de itens.

No terceiro momento, considerando-se a revisão do primeiro, recria-se as definições do apoio tutor, institucional, familiar, financeiro e dos componentes confiança e satisfação do modelo ARCS, de Keller (1987), com o objetivo de melhor operacionalizá-las.

l) Apoio tutor: redefine-se como um provedor do esclarecimento de dúvidas, um gerador do senso de comunidade, instrutor de atividades individuais ou em grupo do curso, condutor significativo com tecnologias interativas e contextualizadas para o sucesso da aprendizagem do estudante durante seus estudos na modalidade de ensino à distância.

m) Apoio institucional: pavimenta caminhos, que consistem de recursos tecnológicos e infraestrutura administrativa centralizada, para os estudantes utilizarem e concluirem seus programas de curso.

n) Apoio familiar: nivel de apoio que os alunos recebem de seus familiares e amigos para concluir o programa de curso.

o) Apoio Financeiro: mudanças nas circunstâncias da vida do discente durante o processo de formação, especificamente relacionadas à dificuldades financeiras, que o obriga a abandonar seu programa de curso.

p) Confiança: fornecem senso de autoestima, expectativa positiva e capacidade de sucesso em tarefas desafiadoras.

q) Satisfação: estratégias que apoiam os esforços dos estudantes e fazem com que sintam-se bem sobre suas realizações.

Após o processo de recriação das definições, revisou-se cada um dos itens, bem como sua representatividade e relevância para o constructo. Refinamos, reformulamos e padronizamos os itens. Em seguida, buscou-se selecionar, na literatura, a escala de verificação para mensuração de construtos de múltiplos itens em concordância de estudantes a determinadas afirmações, relacionadas aos seus respectivos construtos.

Tomou-se o conceito de Finkelstein (2003. 2009) e Mari (1996, 1999), que afirmam que a mensuração (ou medição) é definida como atribuição de símbolos, preferencialmente numéricos, à propriedade dos objetos que se deseja medir. Esses símbolos são direcionados a quantificar ou classificar determinadas características. Sendo assim, a medição é um processo de representação, relacionando algum aspecto do mundo real com sistemas simbólicos. Assim, identificou-se a escala Likert, com cinco pontos no Quadro 2, variando de um (discordo totalmente) a cinco (concordo totalmente), uma ferramenta essencial para capturar e facilitar a manipulação de dados.

Quadro 2 - Escala tipo Likert com cinco pontos.

\begin{tabular}{|l|l|l|l|l|}
\hline $\begin{array}{l}\text { Discordo } \\
\text { totalmente }\end{array}$ & Discordo & Indiferente & Concordo & $\begin{array}{l}\text { Concordo } \\
\text { totalmente }\end{array}$ \\
\hline 1 & 2 & 3 & 4 & 5 \\
\hline
\end{tabular}

Fonte: Elaborado pelos autores (2019).

\section{Revisão pré-piloto do instrumento de pesquisa}

O instrumento de pesquisa foi revisado por dois estatísticos. Os comentários desses profissionais serviram de base para manter a ordem conceitual, correção gramatical e reformulação, itens com palavras claramente favoráveis/desfavoráveis.
Considerando-se os comentários dos especialistas estatísticos e o estudo de Lee e Choi (2011), que tem um vasto conteúdo sobre variáveis preditoras de evasão posteriores a admissão de estudantes na EaD, reavaliou-se o constructo "apoio acadêmico" e "apoio financeiro" e adicionou-se dois itens, um em cada constructo, com o objetivo de 
coletar mais informações a respeito do apoio da instituição e do apoio da fonte do investimento feito, totalizando, assim, 24 itens ao todo. Apre- senta-se, no Quadro 3, os elementos acionados e a nova ordem numérica, consolidando-se, assim, os itens do instrumento de pesquisa.

Quadro 3 - Constructos e itens consolidados do instrumento de pesquisa.

\begin{tabular}{|c|c|c|}
\hline Constructo & $N^{0}$ item & Descrição do item \\
\hline \multirow[t]{2}{*}{ Apoio acadêmico } & 1 & O suporte quando prestado pelo tutor foi adequado. \\
\hline & 2 & O suporte quando prestado pela instituição foi adequado. \\
\hline \multirow[t]{2}{*}{ Apoio familiar } & 3 & $\begin{array}{l}\text { Minha familia me entende muito bem, embora eu passe pouco tempo com } \\
\text { eles por causa do curso EaD. }\end{array}$ \\
\hline & 4 & $\begin{array}{l}\text { No geral, meu grupo de apoio de familiares e amigos me incentiva a } \\
\text { concluir meu programa de estudos. }\end{array}$ \\
\hline \multirow[t]{2}{*}{ Apoio financeiro } & 5 & $\begin{array}{l}\text { Às vezes me pergunto se a minha formação vale realmente todo o } \\
\text { investimento que eu fiz. }\end{array}$ \\
\hline & 6 & $\begin{array}{l}\text { Mesmo diante de algumas dificuldades financeiras durante a minha } \\
\text { formação, não penso em desistir do curso. }\end{array}$ \\
\hline \multirow{3}{*}{$\begin{array}{l}\text { Motivação } \\
\text { - subescala } \\
\text { atenção }\end{array}$} & 7 & O material didático e conteúdo interativo deste curso são atraentes. \\
\hline & 8 & $\begin{array}{l}\text { A forma como a informação é estruturada, contextualizada e organizada no } \\
\text { meu ambiente virtual de aprendizagem ajudou a manter a minha atenção. }\end{array}$ \\
\hline & 9 & $\begin{array}{l}\text { A qualidade da metodologia educacional oferecida no curso foi suficiente } \\
\text { para manter a minha atenção. }\end{array}$ \\
\hline \multirow{4}{*}{$\begin{array}{l}\text { Motivação - } \\
\text { subescala } \\
\text { relevância }\end{array}$} & 10 & $\begin{array}{l}\text { O conteúdo do material didático deste curso foi relevante para os meus } \\
\text { interesses. }\end{array}$ \\
\hline & 11 & O conteúdo deste curso relaciona-se com as minhas expectativas e metas. \\
\hline & 12 & Os benefícios pessoais deste curso são claros para mim. \\
\hline & 13 & $\begin{array}{l}\text { As instruções que recebi na ambientação foram úteis, me auxiliaram no } \\
\text { início e na condução do curso que escolhi. }\end{array}$ \\
\hline \multirow{4}{*}{$\begin{array}{l}\text { Motivação - } \\
\text { subescala } \\
\text { confiança }\end{array}$} & 14 & $\begin{array}{l}\text { Depois de ler as informações introdutórias do curso, sinto-me confiante do } \\
\text { que vou aprender com este curso. }\end{array}$ \\
\hline & 15 & Recebi feedback suficiente para saber como estava indo bem no curso. \\
\hline & 16 & Eu senti confiança para aprender o conteúdo e ter sucesso no curso. \\
\hline & 17 & $\begin{array}{l}\text { Quando olhei pela primeira vez para este curso, tive a impressão de que } \\
\text { seria fácil para mim. }\end{array}$ \\
\hline \multirow{3}{*}{$\begin{array}{l}\text { Motivação - } \\
\text { subescala } \\
\text { satisfação }\end{array}$} & 18 & Gostei muito de estudar este curso. \\
\hline & 19 & $\begin{array}{l}\text { O feedback após a correção de provas e trabalhos, me estimulou e ajudou- } \\
\text { me a sentir recompensado pelo meu esforço. }\end{array}$ \\
\hline & 20 & Sinto-me satisfeito com o que recebi deste curso. \\
\hline Sexo & 21 & Selecione seu Sexo: ( ) Masculino ( ) Feminino \\
\hline Idade & 22 & $\begin{array}{l}\text { Qual a sua idade? ( ) } 19 \text { ou menos ( ) } 20-24 \\
\begin{array}{llll}\text { ( ) } 25-29 & \text { ( ) } 30-34 & \text { ( ) } 35-39 & \text { ( ) } 40-44 \\
\text { ( ) } 45-59 & \text { ( ) } 50-54 & \text { ( ) } 55 \text { ou mais }\end{array}\end{array}$ \\
\hline Educação & 23 & $\begin{array}{l}\text { Qual é o seu maior grau de escolaridade antes de entrar no curso de } \\
\text { graduação? } \\
\text { ( ) Diploma do ensino médio （）EJA（）PROEJA } \\
\text { ( ) Licenciado ou de dois anos（） Diploma de bacharel }\end{array}$ \\
\hline
\end{tabular}


Quadro 3 - Constructos e itens consolidados do instrumento de pesquisa.

\begin{tabular}{|c|c|c|}
\hline Constructo & $N^{\circ}$ item & Descrição do item \\
\hline Situação trabalho & 24 & $\begin{array}{l}\text { Trabalhas quantas horas por semana? } \\
\begin{array}{llll}\text { ( ) Menos de } 20 \quad(\quad) 20 \text { a } 29 & \text { ( ) } 30 \text { a } 39 \\
\text { ( ) } 40 \text { a } 49 & \text { ( ) } 50 \text { ou mais } & \end{array}\end{array}$ \\
\hline
\end{tabular}

EaD: ensino à distância; EJA: educação de jovens e adultos; PROEJA: Programa de Integração da Educação Profissional ao Ensino Médio na Modalidade Educação de Jovens e Adultos.

Fonte: Elaborado pelos autores (2019).

\section{Considerações finais}

Apesar da expansão da modalidade de ensino à distância em termos globais, por meio de recursos tecnológicos, que favorecem o ensino-aprendizagem e a qualificação contínua, a alta taxa de evasão de estudantes tem sido motivo de preocupação dos administradores, gestores e pesquisadores da educação. De maneira geral, a literatura sugere que o comportamento da evasão de estudantes ocorre em função de vários motivos ao longo do seu programa de curso na graduação na modalidade à distância

O instrumento desenvolvido neste estudo teve como finalidade coletar informações sobre o que afeta a decisão de abandonar o curso de estudantes do ensino superior à distância. Nesse sentido, o foco da pesquisa foi dirigido para o apoio acadêmico recebido pelos estudantes, as mudanças nas circunstâncias da vida durante o processo de formação, aos aspectos motivacionais, experiência de aprendizagem em geral e caracteristicas individuais. A falta de instrumentos padronizados, que afiram, especificamente, os motivos potenciais sobre o que afeta a decisão de abandonar o curso dos discentes do ensino superior à distância, justificou o desenvolvimento deste estudo. Mesmo com a limitação, no tocante aos números, de juizes especialistas, espera-se que este estudo sirva de base para novas pesquisas e ampliação de conhecimento, para testar motivos potenciais que afetam a decisão de abandonar o EaD de estudantes. Como sugestões para estudos futuros, cita-se uma maior quantidade de juízes especialistas, para identificação, análise e ampliação de relevâncias das solicitações de apoio à construção do instrumento.

\section{Referências}

Ambiel, R. A. (2015). Construção da Escala de Motivos para Evasão do Ensino Superior. Avaliação Psicológica. Interamerican Journal of Psychological Assessment, 14(1), 41-52. http://doi.org/10.1568g/ap.2015.1401.05

Balaban-Sali, J. (2008). Designing motivational learning systems in distance education. The Turkish Online Journal of Distance Education, 9(3).

Bean, J. P.; Metzner, B. S. (1985). A conceptual model of nontraditional undergraduate student attrition. Review of Educational Research, 55(4), 485-540. https://doi. org/10.3102\%2F00346543055004485

Brown, K. M. (1996). The role of internal and external factors in the discontinuation of off-campus students. Distance Education, 17(1), 44-71. https://doi. org/10.1080/0158791960170105

Costa, O.; Gouveia, L. (2019). Educação superior a distância: fatores preditores da evasão anteriores a admissão de estudantes. Revista Educação Em Questão, 57(51). https://doi.org/10.21680/19811802.2019v57n51|D15671

Diniz, A. M.; Almeida, L. S. (2005). Escala de Integração Social no Ensino Superior (EISES): metodologia de construção e validação. Análise Psicológica, 23(4), 461476. https://doi.org/10.14417/ap.562

Finkelstein, L. (2009). Widely-defined measurement: An analysis of challenges. Measurement, 42, 1270-1277. https://doi.org/10.1016/j.measurement.2009.03.009

Finkelstein, L. (2003). Widely, strongly and weakly defined measurement. Measurement, 34, 39-48. https:// doi.org/10.1016/S0263-2241(03)00018-6

Fior, C. A.; Mercuri, E.; Almeida, L. D. S. (2011). Escala de interação com pares: construção e evidências de validade para estudantes do ensino superior. Psico-USF, 16(1), 11-21. https://doi.org/10.1590/S141382712011000100003

Fior, C. A.; Mercuri, E.; Silva, D. (2013). Evidências de validade da Escala de Envolvimento Acadêmico para universitários. Avaliação Psicológica, 12(1), 8189. Retirado de: http://pepsic.bvsalud.org/scielo. php? script=sci_arttext\&pid $=$ S1677-04712013000100011 
Holder, B. (2007). An investigation of hope, academics, environment, and motivation as predictors of persistence in higher education online programs. The Internet and Higher Education, 10(4), 245-260. https:// doi.org/10.1016/j.i.heduc.2007.08.002

Huang, B. E.; Hew, K. F. (2016). Medindo o nível de motivação dos alunos em grandes cursos on-line abertos, International Journal Information and Education Technology. 6(10), 759-764

Jun, J. (2005). Understanding dropout of adult learners in e-learning (Tese de Doutorado). University of Georgia.

Keller, J. M. (2010). Motivational design for learning and performance: the ARCS model approach. New York, NY: Springer

Keller, J. M. (1987). Strategies for stimulating the motivation to learn. Performance \& Instruction, 26(8), 1-7. https://doi.org/10.1002/pfi.4160260802

Kemp, W. C. (2002). Persistence of adult learners in distance education. The American Journal of Distance Education, 16(2), 65-81. https://doi.org/10.1207/ S15389286AJDE1602_2

Lee, Y.; Choi, J. (2011). A review of online course dropout research: implications for practice and future research. Educational Technology Research and Development, 59(5), 593-618. http://doi.org/10.1007/s11423-010$9177-y$

Levy, Y. (2007). Comparing dropouts and persistence in e-learning courses. Computers and Education, 48(2), 185-204. http://doi.org/10.1016/j.compedu.2004.12.004

Loorbach, D.; Frantzeskaki, K.; Huffenreuter, R. L. (2015). Transition management: taking stock from governance experimentation. J Corp Citizenship, 58, 48-66. http:// doi.org/10.9774/GLEAF.4700.2015.ju.00008

Mansfield, M.; O'leary, E.; Webb, S. (2011). Retention in higher education: faculty and student perceptions of retention programs and factors impacting attrition rates. Relatório de pesquisa apresentado a School of Education Indiana University. South Bend, 2011. Retirado de: http://files.eric.ed.gov/fulltext/ED521416.pdf

Mari, L. (1999). Notes towards a qualitative analysis of information in measurement results. Measurement, 25(3), 183-192. http://doi.org/10.1016/S0263-2241(99)00002-0

Mari, L. (1996). The meaning of "quantity" in measurement. Measurement, 17(2), 127-138. http://doi. org/10.1016/0263-2241(96)00022-X

Mill, D. (2012). Docência Virtual: Uma visão critica. Campinas. Papirus.

OLC. (2015). The drive behind the Online Learning Consortium. Online Learning Consortium. Retirado de: https://onlinelearningconsortium.org/about/qualityframework-five-pillars/

Packham, G.; Jones, P.; Miller, C.; Thomas, B. (2004) E-learning and retention: key factors influencing student withdrawal. Education + Training, 46(6/7), 335-342. https://doi.org/10.1108/00400910410555240
Perry, B.; Jeannette, B.; Dean, C.; Margaret, E. (2008). Why do students withdraw from online graduate nursing and health studies education? Journal of Educators Online, 5(1), 1-17. http://doi.org/10.9743/JEO.2008.1.2

Pierrakeas, C.; Xenos, M.; Panagiotakopoulos C.; Vergidis, D. (2004). A comparative study of dropout rates and causes for two different distance education courses. International Review of Research in Open and Distance Learning, 5(2), 1-15. Retirado de: http://www.irrodl.org/ index.php/irrodl/article/view/183/265

Park, J. H. (2007). Factors related to learner dropout in online learning. Americas of the Academy of Human Resource Development, Indianapolis.

Park, J. H.; Choi, H. J. (2009). Factors influencing adult learners' decision to drop out or persist in online learning. Educational Technology \& Society, 12(4), 207-217.

Parker, A. A. (1999). A study of variables that predict dropout from distance education. International Journal of Educational Technology, 1(2), 1-10. Retirado de: https:// www.learntechlib.org/p/90640/

Pedue, T. (1999). Cherokee women: Gender and culture change, 1700-1835. Lincoln: University of Nebraska Press.

Prieto, A. J. (1992). A method for translation of instruments to other languages. Adult Education Quarterly, 43(1), 1-14. https://doi.org/10.1177\%2F0741713692043001001

Schleich, A. L. R.; Polydoro, S. A. J.; Santos, A. A. A. (2006). Escala de satisfação com a experiência académica de estudantes do ensino superior. Avaliação Psicológica, 5(1), 11-20. Retirado de: http://pepsic.bvsalud.org/scielo. php? script=sci_arttext\&pid=S1677-04712006000100003

Shelton, K. (2011). A review of paradigms for evaluating the quality of online education programs. Online Journal of Distance Learning Administration, 4(1). Retirado de: http://www.westga.edu/ distance/ojdla/spring141/ shelton141.html

Su, J. (2016). Successful Graduate Students Perceptions of Characteristics of Online Learning Environments (Dissertação de Mestrado). University of Tennessee.

Tello, S. F. (2007). An analysis of student persistence in online education. International. International Journal of Information and Communication Technology Education, 3(3), 47-62. http://doi.org/10.4018/jicte.2007070105

\section{Oberdan Santos Da Costa}

Doutorando em Ciência da Informação pela Universidade Fernando Pessoa, em Porto, Portugal. Possui mestrado em Gestão de Empresas pela Universidade Lusófona de Humanidades e Tecnologias, em Lisboa, Portugal. MBA Executivo em Gestão Empresarial pelas Faculdades de Ciências Gerenciais da Bahia. Especialização em Formação de Consultores Organizacionais (FCO) pelo ISAN-FGV. Especialização em gestão empresarial pelo ISAN-FGV. 
12/12 Educação, Porto Alegre, v. 44, n. 2, p. 1-12, maio-ago. 2021 | e-34601

\section{Luis Borges Gouveia}

Professor catedrático na Universidade Fernando Pessoa.

\section{Luis Simoes da Cunha}

Professor auxiliar no Instituto Superior Miguel Torga.

\section{Endereço para correspondência}

Universidade Fernando Pessoa

Praça de 9 de Abril, 349

4249-004

Porto, Portugal

Os textos deste artigo foram revisados por Zeppelini Publishers e submetidos para validação do(s) autor(es) antes da publicação. 